\title{
Abstracts from the twenty-third meeting of the pancreatic society of Great Britain and Ireland at the Village Hotel, Leeds, UK
}

Johnson, C D ; Ammori, B J ; Davides, D ; Vezakis, A ; Larvin, M ; McMahon, K J ; Powell, J J ; Hill, G ; Storey, S ; Murchison, J ; Fearon, K ; Ross, J ; Siriwardena, A K ; Pearce, N W ; Johnson, C D ; Smithies, A M ; Sargen, K ; Demaine, A G ; Kingsnorth, A N ; Ammori, B J ; Becker, K L ; Kite, P ; Barclay, G R ; Snider, R H ; Nylen, E S ; White, J C ; Martin, I G ; Larvin, M ; McMahon, M J ; Raraty, M G T ; et al

DOI: https://doi.org/10.1007/bf02925972

Posted at the Zurich Open Repository and Archive, University of Zurich ZORA URL: https://doi.org/10.5167/uzh-156260

Journal Article

Published Version

Originally published at:

Johnson, C D; Ammori, B J; Davides, D; Vezakis, A; Larvin, M; McMahon, K J; Powell, J J; Hill, G; Storey, S; Murchison, J; Fearon, K; Ross, J; Siriwardena, A K; Pearce, N W; Johnson, C D; Smithies, A M; Sargen, K; Demaine, A G; Kingsnorth, A N; Ammori, B J; Becker, K L; Kite, P; Barclay, G R; Snider, R H; Nylen, E S; White, J C; Martin, I G; Larvin, M; McMahon, M J; Raraty, M G T; et al (1999). Abstracts from the twenty-third meeting of the pancreatic society of Great Britain and Ireland at the Village Hotel, Leeds, UK. International journal of gastrointestinal cancer, 25(3):229-250.

DOI: https://doi.org/10.1007/bf02925972 


\section{Abstracts from the Twenty-Third Meeting of the Pancreatic Society of Great Britain and Ireland at the Village Hotel, Leeds, UK}

November 26-27, 1998

Transmitted by Mr. C. D. Johnson 
The Pancreatic Society gratefully acknowledges the following for supporting this meeting:

The British Journal of Surgery Society

Astra Pharmaceuticals Ltd.

British Biotech

Cook UK Ltd.

Eli Lilly and Co. Ltd.

Pentax UK Ld.

Solvay Healthcare Ltd.

Pharma Nord UK Ltd.

Biotrin International

Wyeth Laboratories

Janssen-Cilag Ltd.

Knoll Pharmaceuticals 
Laparoscopic cholecystectomy: Are patients with biliary pancreatitis at increased operative risk?

Ammori BJ, D Davides, A Vezakis, Larvin M. McMahon $M J$

Leeds Institute for Minimally Invasive Therapy, The General Infirmary, Leeds, UK

Introduction: Previous reports of laparoscopic cholecystectniny (LC) in patients with binary pancreatitis suggested increased operative difficulty, high rates of conversion, longer postoperative hospital stay, and greater morbidity and mortality. Aims: To compare operative risk in patients undergoing LC for binary pancreatitis with those undergoing LC for other causes. Material and Methods: Between 1990 and 1997, 892 consecutive patients underwent LC, in keeping with a policy of "LC for all patients". Data were collected prospectively. LC was performed for binary pancreatitis in 63 patients (Group I) and for other causes in 829 patients (Group II). Results: Patients with binary pancreatitis were significantly older (median age 57 vs 50 years, $p=0.009$ ), with greater co-morbidity (ASA III/IV $24 \%$ vs $11 \%, p<0.02)$ included more males $(41 \%$ vs $22 \%, p<0.01)$ and experienced a shorter symptom duration (median 5 vs 12 months, $p=0.008$ ). The groups were comparable with respect to the frequency of previous abdominal surgery, acute inflammation of the gallbladder, the frequency of bile duct calculi detected at intraoperative cholangiography $(8 \%$ vs $6 \%)$ and urgent or elective operation. Moderate to severe adhesions were significantly more frequent after binary pancreatitis ( $46 \%$ vs $29 \%, p<0.05$ ). No significant differences were observed between the two groups with respect to intraoperative (1.5\% Group I vs $3.5 \%$ Group II) or postoperative complications ( $10 \%$ vs $8 \%$ ), conversion rate (0 vs $2.7 \%$ ) or duration of operation (median 92 vs 85 minutes). Conclusion: Despite increased age and co-morbidity and more frequent adhesions, our data showed no evidence that intraoperative or postoperative complications were more frequent in patients with binary pancreatitis than other patients undergoing LC.

Enteral nutrition does not modify the enrly infammatory response in acute pancreatitis.

JJ Powell, G Hill, S Storey. J Murchison, K Fearon, J Ross \& AK Sirrwardena.

University Department of Surgery, Royal Infirmary of Edinburgh.

Introduction Several studies report a benefit from early enteral nutrition (EEN) in acute pancreatitis. Although EEN is thought to reduce the early inflammatory response there is littic evidence to support this paradigm in acute pancreatitis. We report an interim analysis of a randomised controlled trial of EEN in severe acute pancreatitis, focusing on markers of the inflammatory response.

Methods Patients with predicted severe acute pancreatitis were randomised to receive EEN or conventional treatment alone. The inflammatory response was quantified using measurements of protein serum interleukin 6 (IL6), soluble tumour necrosis factor receptor I (sTNFR) and C-reactive (CRP). Value are mean (standard error of the mean).

Results

\begin{tabular}{|c|c|c|c|c|}
\hline & & IL6 $\mathrm{pg} / \mathrm{ml}$ & STNFR $\mathrm{pg} / \mathrm{mL}$ & CRP mg/L \\
\hline \multirow{3}{*}{$\begin{array}{l}\text { Enteral nutrition } \\
\qquad n=13\end{array}$} & Admission & $266(67)$ & $3018(370)$ & $17 !(34)$ \\
\hline & Day 2 & $143(65)$ & $2908(266)$ & $232(30)$ \\
\hline & Day 4 & $64(30)$ & $2930(267)$ & $180(25)$ \\
\hline \multirow{3}{*}{$\begin{array}{c}\text { Conventional } \\
n=14\end{array}$} & Admission & $732(377)$ & $2459(228)$ & $238(36)$ \\
\hline & Day 2 & $358(236)$ & $2626(313)$ & $273(29)$ \\
\hline & Day 4 & $127(63)$ & $2602(338)$ & $213(30)$ \\
\hline \multicolumn{2}{|c|}{ And) sis of vanance (enteral vs conventional } & $p=281$ & $p=.559$ & $p=624$ \\
\hline
\end{tabular}

Conclusions Our results demonstrate that EEN does not modify the early inflammatory response in severe acute pancrearitis. 
Antibiotic use during the study of lexipa fant in severe acute pancreatitis

Pearce NW and Johnson C.D.

University Surgical Unit Southampton

290 patients participated in a multi-centre, double-blind, randomised placebo controlled, parallel group study of Lexipafant in severe acute pancreatitis.

Antibiotic use was recorded prospectively during the study and was similar in both the Lexipafant and placebo groups ( $95 / 148$ and $99 / 138$ respectively) of whom most were on antibiotics at recruitment (71/95 and 75/99). The majority received a cephalosporin (76/95 and 78/99). Patients were divided into three groups:- patients taking antibiotics at recnitment were termed the "early" group, patients starting antibiotics later were called the "delayed" group and thirdly the "no antibiotic" group. There were more local complications in the "delayed" group (16/48, 33\%) than in the "carly" $\left(38 / 146,26 \%\right.$, n.5.) or "no antibiotic" groups $\left(10 / 92,11 \%, x^{2}\right.$ $p<0.01)$. Patients with a local complication were more likely to develop local sepsis in the "delayed" $\left(9 / 16,56 \%, \chi^{2} p<0.001\right)$ or "no antibiotic" groups $\left(4 / 10,40 \%, \chi^{2}\right.$ $\rho<0.05)$ than in the "early" group $(5 / 38,13 \%)$ - Patients with necrosis were less likely to develop local infection in the "early" group than in the combined "delayed" and "no antibiotic" groups $\left(5 / 27 \times 13 / 25 \chi^{2} p<0.01\right)$. Patients with sepsis were less likely to die if they were in the "early" antibiotic group $\mathrm{Cm}$ if they were in the combined "delayed" or "no antibiotic" groups (3/8 v 8/9 $p<0.05$ Fisher's exact test)

Patients with predicted severe acute pancreatitis receiving early antibiotics are less likely to develop septic local complications and are less likely to die from septic complications than patients receiving delayed or no antibiotic treatment.

Investigation of the IL-1 gene cluster and its association with acute pancreatitis

\section{A. M. Smithies, K. Sargen, A, G, Demaine, A. N. Kingsnorth}

Departments Molecular Medicine and Surgery, Plymouth Postgraduate Medical School, Plymouth PLA 8AA, UK

Recent studies have implicated the $\mathbb{L}-1$ gene cluster in acute pancreatitis, an inflammatory condition of the pancreas. A penta-allelic polymorphism is known to exist in intron 2 of the IL-Ira gene in which there are a variable number of tandem repeat units each $86 \mathrm{bp}$ long. In addition, a biallelic polymorphism is present in exon 5 of the IL- $1 \beta$ gene which involves a $C$ to $T$ substitution. The ain of this study was to investigate these polymorphisms in acute pancreatitis. The genotype and allele frequencies were determined in patients $(n=125)$ and healthy controls $(n=150)$ using the polymerase chain reaction. Patients were categorised according to actiology (alcohol, idiopathic, gallstone), severity (mild/severe) and organ failure scores (OFS $\geq>2$, OFS $\geq 3$, OFS $\geq 4$ ). There was an increased frequency of allele $I$ of the ILIra polymorphism in idiopathics compared to controls $(82.4 \%$ vs $63 \%, p<0.01)$. No association was found with any other aetiological group. The frequency of allele I was also significantly increased in severe cases compared to mild cases $(81.9 \%$ vs $68.2 \%, p<0.05$ ), however, the increase did not correspond to the organ failure scores. IL-1ra may determine severity of acute pancreatitis and susceptibility to idiopathic acute pancreatitis. No association was found between $I L-1 \beta$ and the disease. 
Aminoprocalcitogin in acute pancreatitis: a better predictor of severity and correlates with endotoxaemia, subsequent sepsis and death

Ammori BJ, Becker $K L^{* *}$, Kite $P^{*}$, Barclay GR***, Snider RH**, Nylen ES**, White JC**, Martin IG, Larvin M, MCMahon MJ

Academic Surgical Unit and "Department of Microbiology, The General Infirmary, Leeds, UK. *George Washington University, Washington, DC, USA, ***Blood Transfusion Services, The Royal Infirmary, Edinburgh.

Introduction: Early prediction of disease severity in patients with acute pancreatitis is important for optimal clinical managernent. Aminoprocalcitonin (nPCT), a 57 amino acid peptide, is a sensitive marker of inflammation and infection. AIMS: To investigate the role of an assay for serum $\mathrm{nPCT}$ as an indicator of severity in patients with acute pancreatitis and to relate serum nPCT changes to endotoxaemia, sepsis and death. Methods: APACHE-II scores and Serum nPCT and endotoxin were determined within 24 hours of admission with acute pancreatitis. $C$-reactive protein (CRP) was measured daily. nPCT concentration was determined by radioimmunoassay. Attacks were classified as mild or severe according to the Atlanta criteria. Results: There were 69 patients: 55 mild and 14 severe. Severe attacks were associated with significantly greater 24 hours APACHE-II scores (median 11 vs 8,p 0.02) and peak CRP concentrations (median 202 vs 98 , $p<0.001)$ compared with mild attacks. nPCT concentrations on admission were significantly greater in severe attacks compared with mild (median 783 vs 154 , $p=0.016$ ), and in those which developed septic complications, fatal or not, compared with other attacks (median 1537 vs 165, $\mathrm{p}=0.025$ ). By receiver operating characteristic analysis, the best cut-off level for predicting severe attacks was 200 $\mathrm{pg} / \mathrm{ml}$, with higher sensitivity and specificity compared with admission APACHE-11 of $>7$ ( $75 \%$ v $69 \%$ and $75 \%$ v $45 \%$ respectively). A positive correlation was observed between admission nPCT senum levels and endotoxaemia $(r=0.5$,

$p<0.00$ l). Conclusion: Serum nPCT $>200 \mathrm{pg} / \mathrm{ml}$ at the time of admission gave a better prediction of severe acute pancreatitis compared with APACHE-II scores. 
Cytosolic calcium rise from hyperstimulation or store depletion induces vacuolisation and enzyme activation in mouse pancreatic acinar cells.

MGT Raraty't, JB Ward'.2, C. Vaillant', JP Neoptolemos', R Sutton', OH Petersen ${ }^{2}$

Departments of Surgery ${ }^{1}$, Physiology ${ }^{2}$, and Veterinary Preclinical Sciences ${ }^{3}$, University of Liverpool, Liverpool, UK.

Background: The intracellular event that initiates acute pancreatitis is unknown. This study tested the hypothesis that acute pancreatitis is triggered by abnormal elevations of acinar free ionised cytosolic calcium $\left(\left[\mathrm{Ca}^{2+}\right] \mathrm{j}\right)$

Methods: Isolated mouse pancreatic acinar cells with or without pre-loading of the specific calcium chelator BAPTA were incubated with $10 \mathrm{nM}$ cholecystokinin (CCK, for hyperstimulation), $2 \mu \mathrm{M}$ thapsigargin (for calcium store depletion) or neither for 60 minutes. Calcium measurements were performed using fura-2. Image analysis of light and electron microscope sections was used to quantify the degree of cytoplasmic vacuolisation as a marker of cell damage. Cleavage of the specific fluorescent substrate for trypsin, bis(isoleucine-proline-arginine) thodamine 110 $\left((I P R)_{2}-R ! 10\right)$, was measured by fluorescence after excitation at $485 \mathrm{~nm}$ as a marker for intracelludar enzyme activation.

Results: Cholecystokinin and thapsigargin both induced increases in $\left[\mathrm{Ca}^{2+}\right]$; of $724 \pm 30 \mathrm{nmol} /$ (mean- + S.E.) and $223 \pm 11 \mathrm{nmol} / \mathrm{L}$ respectively, and increases in vacuolisation from $3.0 \pm 0.31$ vacuoles/cell and $1.1 \pm 0.21 \%$ cell area (in control cells) to $9.9 \pm 0.55$ and $4.3 \pm 0.34 \%$ (CCK) and to $13.8 \pm 0.74$ and $5.5+-0.43 \%$ (thapsigargin). Preloading with BAPTA significantly attenuated the $\left[\mathrm{Ca}^{2+}\right]_{i}$ increases to $216 \pm 28 \mathrm{nmo} / \mathrm{L}(C C K)$ and $47 \pm 6 \mathrm{nmol} / \mathrm{L}$ (thapsigargin), and also markedly reduced vacuolisation to $4.0 \pm 0.38$ vacuoles/cell and $1.1 \pm 0.13 \%$ (CCK) and to $7.4 \pm 0.69$ and $2.0 \pm 0.24 \%$ (thapsigargin). In parallel with the appearance of vacuoles, there was a marked increase in the rate of cleavage of (IPR $h_{2}-R / 10$

Conclusions: These data indicate that abnormal elevations of $\left[\mathrm{Ca}^{2+}\right]$, trigger intracellular abnormalities characteristic of acure pancreatitis including acinar cell vacuolisation and intracellular enzyme activation, and suggest that such elevations in $\left[\mathrm{Ca}^{2+}\right]$, may initiate acute pancreatitis.

Mutations in cationic trypsinogen cause hereditary pancreatitis in patients originating in the North East of England

J.E. Creighton。 *R. Lyall。*D.1. Wilson, *A. Curtis and R.M. Chamley

Dept. of Hepato-Pancreatico-Biliary Surgery, Freeman Hospital, Newcastle and - Dept of Human Genetics, Northern Region Genetics Service

Hereditary pancreatitis (HP) has been linked to mutations in cationic typsinogen. AG to $A$ transition mutation in exon 3 has been shown to cause the disease in families originating from the USA and Italy. This mutation creates a novel recognition site for Afl III, a restriction endonuclease. Other mutations in exon 2 have also been found.

Seven families with HP have been identified by this unit. DNA was extracted from venous blood, and exon 3 of cationic trypsinogen amplified by the polymerase chain reaction. The PCR product was digested with Afl III and fragments sized by agarose gel electrophoresis. Haplotype analysis of the families was carried out. Other exons were also amplified and then sequenced.

Three of the families carried the mutation in exon 3. Of these, two carried the same haplotype, suggesting a common ancestor. Three of the remaining families demonstrated an $A$ to $T$ mutation in exon 2 on sequencing.

A single mutation in exon 3 of cationic trypsinogen is a common cause of hereditary pancreatitis in patients from the North East of England. Further mutations are being found. 
Trypsinogen mutations in families with Hereditary Pancreatitis in the UK \& Ireland

N Howes, 'S Rutherford, ${ }^{3} F$ McRonald, 'I Ellis, ${ }^{2} D$ Whitcomb, ${ }^{4} R$ Mountford, ${ }^{3} J P$ Neoplolemos, for the UK and Ireland consortium of EUROPAC

Dept, of Surgery' \& Clinical Genetics ${ }^{2}$, Univ. of Liverpool; Dept of Molecular Genetics Women's Hospital Liverpool ${ }^{3}$, \& Dept. of Medicine, Univ. of Pitsburgh"

Causative mutations for Hereditary Pancreatitis (HP) have been identified in the cationic trypsinogen genes at $7 \mathrm{q} 35$, which prevent the auto-inactivation of trypsin and autodigestion of the pancreas. The aims of our study were to identify cationic trypsinogen mutations in UK and lrish families and identify phenotype genotype correlations. After pedigree and epidemiological analysis, members of families with HP were screened for the published cationic trypsinogen mutations. DNA analysis was directed at the R117H mutation, The products wore digested with the restriction enzyme Afll 1 , which recognises the common $G$ :A mutation in $H P$. In families negative for this mutation, the N211 mutation was screened using PCR and restriction digestion. 30 families have been referred. We have tested 18 of these for the $\mathrm{R} 117 \mathrm{H}$ and $\mathrm{N} 211$ mutations. 7 of the families $(\mathrm{N}=17)$ have tested positive for the $\mathrm{R} 117 \mathrm{H}$ mutation and 6 for the $\mathrm{N} 21 \mathrm{I}(\mathrm{N}=0)$ mutation, 5 families $(\mathrm{N}=9)$ had neither mutation. Patients with the $\mathrm{R} 117 \mathrm{H}$ variant developed symptoms at an earlier age $(p=0.03)$, and suffered more attacks $(p=0.07)$ compared to the $N 211$ variant. These early findings are similar to the Midwest Multi-centre Pancreatitis Study Group. Our findings also suggest that additional mutations and or genes are responsible for causing HP. Stats: Mann Whitney U

\section{Earfy experience with laparoscopic pancreatectomy}

Ammori BV, A Vezakis, D Davides, Larvin $M$

Leeds Institute for Minimally Invasive Therapy, The General Infirmary, Leeds, UK

Introduction: Laparoscopic surgery is associated with a reduction in postoperative pain and hospital stay, earlier return to normal activity and improved cosmesis. However; the laparoscopic approach to pancreatic surgery is still evolving. Material and Methods: Between 1993 and 1998, 9 patients underwent laparoscopic distal pancreatectomy for endocrine tumours $(n=-4)$, cystadenoma $(n=3)$, adenocarcinoma $(n=1)$ and chronic pancreatitis $(n=1)$. Results: There were seven females and three males. The median age of patients was 62 years (range 30-83 years). Distal pancreatectomy with splenic preservation was performed in 9 patients, and enucleation in one patient. The ultrasonic dissectors were utilised and facilitated dissection. Nonetheless, the procedure was converted to open pancreatectomy in two patients. The pancreas was divided with a linear stapler in 8 patients and with the harmonic scalpel in one. Median operative time was 300 minutes (range 240-360 minutes). Postoperative pancreatic fistula developed in two patients in whom the pancreatic stump was not oversown, and responded to radiological and supportive management. There were no postoperative deaths Median postoperative hospital stay was 12 days (range 5-60 days). All laparoscopically. treated patients remain well at a median follow up of 24 months (3-36). Conclusion: Laparoscopic pancreatectomy is a technically demanding procedure, but appears feasible and safe. Oversewing of the divided pancreatic stump may reduce the risk of pancreatic fistula. The benefits of the laparoscopic approach remain to be demonstrated. 
Improvements in operative mortality for pancreatico-duodenectomy in the west midlands following the establishment of a specialist unit.

M Manu, SR Bramhall, U Gur, BK Gunson, KJ Le Cornu, AD Mayer, DF Mirza, $P$ MCMaster, JAC Buckels

Liver Unit, Queen Elizabeth Hospital, Birmingham

In a recent West Midlands series of pancreatic resections an operative mortality rate of $27 \%$ was reported with a SYSR of $5 \%$. These results and others, have led to the establishment of specialist units. This unit was established in 1986 and has seen an exponential increase in HPB workload.

A retrospective review was performed of all patients who underwent pancreaticoduodenectomy for tumours between January 1987 and June 1998.

169 pancreatico-duodenectomies were performed, the male to female ratio was 1.65 , the mean age was 59 years, with a 30 day mortality of $6 \%$ and a complication rate of $14 \% .41 \%$ of patients had pancreatic adenocarcinoma, $25 \%$ ampullary, $11 \%$ cholangiocarcinoma, $8 \%$ neuroendocrine and $5 \%$ duodenal adenocarcinoma. One and 5 YSR for pancreatic adenocarcinoma and ampullary carcinoma were $47 \%$ and $6 \%$ and $64 \%$ and $6 \%$ respectively, $40 \%$ of these patients were lymph node and resection margin negative and the one and five YSR in this group was $85 \%$ and $0 \%$.

These results show that referral and concentration of cases to a specialist unit leads to a significant improvement in operative mortality but with very little improvement in SYSR. In the subgroup of patients with early disease one YSR appears to be improved but with no effect on five YSR.

Diagnosis and staging of pancreatic carcinoma: comparison of mangafodipir trisodium (MaDPDP) enhanced MRI and spiral CT

LI King, JRG Bell, J C. Healy, Z Amin, D Predolact, A Wotherspoon*, J Thompson ${ }^{t}$

Departments of Radiology and ${ }^{\dagger}$ Surgery, Chelsea and Westminster Hospital,

Department of *Histopathology, The Royal Marsden Hospital, London

Aim: To compare MnDPDP enhanced MRI and spiral CT in the diagnosis and staging of pancreatic carcinoma.

Methods: Nineteen patients with suspected pancreatic carcinoma were evaluated with $M R I$ and $C T$. Unenhanced $T l / T 2$, and $T 1$ images 20 minutes and 24 hours following infusion of $0.5 \mathrm{ml} / \mathrm{Kg} \mathrm{MnDPDP}$ were obtained at $1.0 \mathrm{~T}$. CT was performed during pancreatic and portal venous phases of enhancement. Scans were independently assessed for the presence of a pancreatic mass. Resectability was determined by evaluating extraglandular extension, vascular involvement, lymphadenopathy, peritoneal spread and hepatic metastases. Imaging and surgical findings were then compared.

Results: Pancreatic adenocarcinoma was diagnosed histologically in 15/19(79\%) patients. MRI and CT both identified 15/15 (100\%) tumours. Clear evidence of non-resectability was found in $6 / 15(40 \%)$ patients at MRI, who did not progress to surgery. CT was equivocal in one of these cases. Nine patients progressed to laparoscopy $(n=2)$ or laparotomy $(n=7)$. Of these 9 patients, resectability was correctly assessed in $7(77.8 \%)$ on MRI and $6(66.7 \%)$ by CT. The overall accuracy for resectability was $86.7 \%$ for MRI and $73.3 \%$ for $C T$. Liver metastases were confidently identified in $3 / 15(20 \%)$ patients on both MRI and CT. Indeterminate liver lesions on CT in two patients were shown to be benign on MRI.

Conclusion: MRI performed better than $C T$ in assessing tumour resectability and evaluating associated liver lesions. There was no difference in tumour detection between $M R I$ and $C T$. 
Endoscopic ultrasonography (EUS) in staging pancreatobiliary neoplasia

SA Norton, D Alderson

Bristol Royal Infurnary

Introduction Most peripancreatic tumours have a poor prognosis. Radical surgery should be avoided for inoperable tumours or if significant survival benefit is unlikely. Current investigations are limited in identifying resectability and lymph node metastases. This study aimed to assess the accuracy of EUS in locoregional staging of pancreatobiliary malignancy compared to existing modalities.

Patients and Methods 41 patients with confirmed pancreatobiliary malignancy were studied. Each underwent EUS in addition to standard pre-operative imaging with trans-abdominal uitrasonography (TUS) and computed tomography (CT). Results were compared to surgical and histological staging.

Results All patients underwent trial dissection, with resection possible in 18/41 cases, the remainder undergoing palliative bypass. TUS could not visualise 21 tumours, so could not be expected to identify vascular invasion. Sensitivity, specificity. PPV and NPV of CT in predicting vascular involvement was $35.7 \%$ (CI 13-65\%), 100\% (CI 85-100\%), 1 (CI 0.45-1) and 0.71 (Cl 0.52-0.86) respectively. The presence of 3 or more of 6 EUS features associated with vascular invasion had sensitivity of $80 \%$ (Cl $44-98 \%$ ), specificity of $94.4 \%$ (CI $73-100 \%)$, PPV of 0.89 ( $\mathrm{Cl} 0.52-1)$, and NPV of 0.89 ( $\mathrm{Cl} 0.67-0.99)$. EUS was unhelpful in large tumours which prevented duodenal intubation, or caused displacement of vessels beyond the range of EUS.

Conclusion EUS can provide more accurate staging of pancreatobiliary tumours in selected cases. Larger studies are required to determine the impact of berter staging on patient outcome.

Overexpression of c-erbB-2, c-erbB-3 and cyclinDi and loss of p53, p16 and p21 do not correlate with progaosis in resected ductal pancreatic cancer

Kawesha $A^{\prime}$. Ghaneh $P^{\prime}$. Evans $J D^{\prime}$, Campbell', Dawiskiba $S^{3}$ Lemoine NR', Andrén-Sandberg $A^{3}$ and Neoptolemos $J P^{\prime}$

Departments of Surgery' and 'Pathology, University of Liverpool, UK; Departments of ${ }^{3}$ Pathology and ${ }^{3}$ Surgery, Lund University, Sweden; 'ICRF, London UK

The pathogenesis of pancreatic cancer bas been associated with overexpression of certain growth factors and their receptors, activation of proto-oncogenes and inactivation of tumour suppressor genes but their role in prognosis is not known. The aim of our study was to use immunohistochemistry to study the expression of cerbB-2, c-erbB-3, cyclin D1, p53, p21 and p16 in paraffin-embedded tissue sections and correlate the findings with pathological parameters and survival. 138 resected ductal adenocarcinomas were studied comprising a consecutive series of patients from Sweden ( $n=69$. median age $=63$ years) and an equal number from the United Kingdom with similar median age. Median survival following resection was 234 days in the Swedish cases and 353 days in the British cases $(p=0.03)$. Results of positive staining was as follows:

$\begin{array}{lllllll}\text { Country } & \text { p53 } & \text { p21 } & \text { p16 } & \text { c-erbB-2 } & \text { c-erbB-3 } & \text { cyclin D1 } \\ \text { UK } & 46 \% & 30 \% & 11 \% & 30 \% & 58 \% & 65 \% \\ \text { Sweden } & 33 \% & 25 \% & 14 \% & 30 \% & 39 \% & 78 \%\end{array}$

Frequency distribution of age, gender, differentiation, tumour size, lymph node status, stage or survival was the same in the presence or absence of the genetic markers studied. In conclusion, the genetic markers studied although of considerable importance are of no prognostic value. (Statistics: Chi Squared Test) 
Tumour suppressor gene therapy in pancreatic cancer

P Ghaneh', M Humphreys', W. Greenhalf', NR Lemoine', JP Neoprolemos'.

'Department of Surgery, Royal Liverpool Hospital, Liverpool, UK

${ }^{2}$ ICRF Molecular Oncology, Hammersmith Hospital, London, UK.

Pancreatic cancers demonstrate high rates of loss of function of the tumour suppressor genes 53 and $p 16^{\mathbb{N} K 4.4}(70 \%$ and $80 \%$ respectively). These genes have a major role in cell cycle control; promoting $G 1$ arrest and $p 53$ dependent apoptosis. The aim of this study was to replace the wild-type (wt) p53 and p16 genes into pancreatic cancer cells and assess the effects on cell growth rates and to evaluate tumour cell killing. Human pancreatic cancer cell lines $\mathrm{BxPc} 3$,

MIAPACA2, Panc1, Capan2, (LOH p53, homozygously deleted/mutated pl6) were used. Adenovirus was used to transfer either wt p53 or wt pl6 alone and in combination. Therapeutic gene transfer was confimed using PCR, $t-P C R$, western blotting and immunofluorescence. Cell counts were used to plot growth curves. FACS analysis was used to assess cell cycle distribution and apoptosis. DNA laddering was used to confirm apoptosis. Transfer of $\mathrm{p} 53$ inhibited cell growth by $78-91 \%$, p 16 by $66-83 \%$ and in combination the median growth inhibition rate was increased. Significant rates of apoptosis was induced by $\mathrm{p} 16$ and $\mathrm{p} 53$ transfer ( $p<0.004, p<0.03$ respectively) and in combination. Adenoviral mediated transfer of p53 and p 16 alone and in combination resulted in significant growth inhibition and cell killing. This indicates a role for tumour suppressor gene therapy in pancreatic cancer.

9 may be better than 3 when it comes to sphincter of Oddi manometry.

EA Stoner, AJK Plotrowicz, DF Evans, CC Ainley.

The GI science research unit, The London and St. Bartholomew's school of Medicine, London.

Catheter design for sphincter of Oddi (SO) manometry has not changed in nearly 20 years. The Lehman (Wilson-Cook, USA) viple lumen perfused catheter is the most widely used. Pressure measurements are made from two of the three lumens. These two lumens open $2 \mathrm{~mm}$ apant in distance and 180 degrees in onentation, at the distal end of the catheter. SO manometry using this catheter sometimes shows differing pressure profiles from each channel, making the interpretation of results difficult and suggesting sphincter asymmetry. Aims: The aim of this study was to assess sphincter asymmetry using a new 9 lumen catheter, designed in conjunction with Medi-plus, UK. Results: The new nine lumen catheter has 8 perfused channels which open at the same level and are orientated uniformly around 360 degrees. The ninth channel is for aspiration. Three shudies have been perfomed so far in patients with suspected SO dysfunction. A station pull through technique was used. All three patients had asymmetric sphincter pressure profiles. In two patients it was marked, with a $110 \mathrm{mmHg}$ pressure difference between the maximum and minimum pressures at one station. Conclusion: The $\mathrm{SO}$ is asymmetric and the new nine lumen catheter is superior in assessing this. 
Raised intact and split pro-insulin with insulin deficiency after islet autotransplantation

SA White, C Pollard, AC Burden, HA Clayton, JE Davies, SM Swift, CN Hales, AR Dennison, NJM London

Department of General Surgery, Leicester General Hospital, Gwendolen Road, Leicester LES 4PW

Simultaneous islet autotransplantation (AT) following total pancreatectomy for chronic pancreatitis (CP) can prevent insulin dependence. In an artempt to explain insulin dependence a prospective analysis of the secretion of insulin, intact proinsulin IPI), 32/33 split pro-insulin (SPI) as well as C-peptide was performed. Overall, 11 consecutive patients having islet AT were assessed. These were compared to patients having $C P$ with either impaired glucose tolerance $(n=6)$ or with normal glucose tolerance $(n=15)$. Patients with NIDDM and non-diabetic patients (controls) without $\mathrm{CP}$ were also evaluated. All patients received an oral ( 75 gram) glucose tolerance test (GTT). The results are tabulated below:

\begin{tabular}{|c|c|c|c|c|c|c|c|}
\hline & $\begin{array}{l}\text { C-pep } \\
\text { pmot }^{-1}\end{array}$ & $\begin{array}{l}\text { Glucoss } 0 \\
\text { min mmo }\end{array}$ & $\begin{array}{l}\text { Glucose } 2 \\
\text { hrs munot" }\end{array}$ & $\begin{array}{l}\text { Insulin } 30 \\
\text { min prnor' }\end{array}$ & $|P| \mathrm{pmol}^{-1^{*}}$ & SPI prnor: & $\begin{array}{l}\text { Ratio: } \\
\text { IPI:SPI }\end{array}$ \\
\hline Controls & 863 & 5.5 & $<7.8$ & 304 & 3.80 & 6.30 & \\
\hline NIDDM & 580 & $>7.8$ & $>11.10$ & 138 & 10.82 & 8.10 & 1.40 \\
\hline $\begin{array}{l}C P(n=15) \\
\text { Nomal } \\
\text { GTT }\end{array}$ & 683 & 4.35 & 4.58 & 387 & 4.20 & 6.78 & 0.81 \\
\hline $\begin{array}{l}\text { CP }(n=6) \\
\text { Impaired } \\
\text { GTT }\end{array}$ & 467 & 7.12 & 10.70 & 101 & 3.55 & 4.25 & 1.02 \\
\hline $\begin{array}{l}\text { Islet AT } \\
(n=11)\end{array}$ & 144 & 7.91 & $\$ 7.55$ & 144 & 0.02 & 30.96 & 0.31 \\
\hline
\end{tabular}

fasting samples. All mean values.

In conclusion, these results deraonstrate insulin deficiency, raised intact and very high $32 / 33$ split pro-insulin in the presence of hyperglycaemia despite C-peptide levels within the normal range in islet autotransplant recipients. Possible explanations include islet damage during the isolation process, non-physiological revascularisation or porto-systemic overspill of islet homones. 
Stented cyst gastrostomy for pancreatic pseudocysts

Sutton $C D$. White $S$, Berry $D P$, Chillistone $D$, Rees $Y$, Dennison $A R$

Department of Surgery, Leicester General Hospital, Gwendolen Road, Leicester LE5 4PW

Background We set out to review a single hepatobiliary surgeons experience of endoscopic treatment of pancreatic pseudocysts. Several methods of endoscopic treatment have been described, the technique used in Leicester General Hospital consists of a combined endoscopic and ultrasound guided percutaneous placement of a double j-stent.

Method Between March 1994 and May 1998, data was refrospectively collected for patients who had undergone endoscopic stenting of a pancreatic pseudocyst. Their case notes were examined and a database constructed.

Results Over the study period, 20 consecutive patients were identified. All patients had undergone a standard technique of combined endoscopic and ultrasound guided percutaneous placement of double J stents, between a pancreatic pseudocyst and the stomach. No patients were lost to follow up which ranged between two months and four years. The age range was between 26 and 84 years. 14 of the pseudocysts were due to acute pancreatitis and 6 were due to chronic pancreatitis. All could be seen to be bulging into the posterior wall of the stomach at endoscopy. Operation length ranged between 30 and 95 minutes and hospital admission ranged between 2 and 77 days. Only two patients suffered complications postoperatively; one being readmitted two weeks following stenting with acute cholecystitis, the other suffering a perforated duodenal ulcer three weeks after stenting. There were two failures early in the series, both due to stent migration, these stents were of a small size ( $4.7 \mathrm{Fr}$ ). Following this, the stent size was increased to at least $7 \mathrm{Fr}$; no further failures occurred. There was no operative mortality for the series.

Conclusion A combined percutaneous and endoscopic cyst-gastrostomy stent is a safe and effective treatment for patients with suitably placed pseudocysts. 


\section{Posters}

1. Feasibility and outcome of early enteral nutrition in severe acute pancreatitis JJ Powell, J Murchison, G Hill, S Storey, K Fearon. J Ross \& AX Siriwardena

2. Endoscopic transgastric drainage of pancreatic pseudocysts and abscesses complicating acute pancreatitis $J$ Cordova \& $T$ Leese

3. Polymorphism of the $\mathbb{L}-10$ gene in patients with acute pancreatitis $K$ Sargen, AG Demaine \& AN Kingsnorth

4. United Kingdom and Ireland survey of antibiotic prophylaxis in acute pancreatitis $J J$ Powell, E Campbell, CD Johnson \& AX Siriwardena

5. Endoscopic ultrasound (EUS) in the diagnosis of pancreatobiliary tumours SA Norton \& D Alderson

6. Serum isoamylase and lipase activities in HIV positive clinical categories MJMJ Deenamode, A Pozniak \& RA Sherwood

7. Pancreatic lipase and amylase in fulminant hepatic failure: an immunohistochemical survey MJMJ Deenamode, B Portmann, R Przemioslo, A Rayner \& RA Sherwood

8. Serum isoamylase and lipase activities in pretern infants MJMJ Deenamode, RM Nicholl. HR Gamsu \& RA Sherwood

9. Audit of deaths from acute pancreatitis in the south east of Scotland 1995-96 Evidence for variation in management? $A C$ de Becux \& $S J$ Nixon

10. Investigation of the effect of hypoxia on matrix metalloproteinase expression in pancreatic cancer cell lines DM Williams, JA Griffin. GM Matthews, JAC Buckels \& SR Bramhall

11. The evolution of a system specific tumour database K le Comu, BK Gunson, SR Bramhall, DF Mizra, AD Mayer, P McMaster \& JAC. Buckels

12. Neutrophil activation in acute pancreatitis - the two hit hypothesis confimed A Steven, CMCKay, S Robertson, S Evans, CR Carter \& CW Imrie

13. Recent time trends in England and Wales in gastric and pancreatic cancer mortality $D$ Filzsimmons, C Osmond, $S$ George \& CD Johnson

14. Minimal access techniques in the management of infected pancreatic necrosis $R$ Carter, CJ McKay \& CW Imrie 
Feasibility and outcome of early enteral nutrition in severe acute pancreatitis.

JJ Powell, J Murchison, G Hill, S Siorey, K Fearon, J Ross \& AK Siriwardena

Introduction Although several small studies have reported a benefit from early enteral nutrition (EEN) in acute pancreatitis the scope, safety and benefits of this technique remain unresolved issues. We therefore report the results of an interim analysis of a single centre randomised controlled trial of EEN in severe acute pancreatitis, focusing on feasibility, tolerance and complications of EEN.

Methods 14 patients were allocated to receive EEN via a nasojejunal feeding tube placed under radiological screening. For each individual, caloric requirement was determined using the Schofield equation. Values are median (range)

Results Tube placement was successful in 12 individuals. Duration of screening was $3.0(0.9-11.9)$ minutes. The median number of tube dislodgement episodes was $1(0-3)$ per person. Tubes remained in place for a median of $2(1-7)$ days. The median number of tube placements was $1(1-3)$ per person. The median proportion of daily caloric requirement supplied by nasojejunal feeding was $21 \%$ ( 0 $100 \%$ ). In 3 patients the volume of feed was limited because of ileus. One patient had probable feed-induced diarthoea. Normal diet was introduced at a median of 5 (4-9) days.

Conclusions Nasojejunal tube placement is feasible but there is a high rate of tube dislodgement. EEN cannot provide all the nutritional support required in severe acute pancreatitis. Our findings are at variance with previous studies therefore justifying continued investigation.

Endoscopic transgastric drainage of pancreatic pseudocysts and abscesses complicating acute paacreatitis

$J$ Cordova and $T$ Leese

Department of Surgery, Royal Lancaster Infirmary

Endoscopic transgastric drainage was introduced at a District General Hospital in 1995 as a new modality for draining pancreatic pseudocysts and abscesses following acute pancreatitis. The technique is employed for manure pseudocysts and abscesses with good adherence to the stomach wall on $\mathrm{CT}$ scan. It is not used if there is significant pancreatic necrotic debris when we prefer an open drainage technique allowing pancreatic necrosectomy at the same time.

Six patients have undergone transgastric drainage for pseudocyst (5) or late abscess in the pancreatic bed following pancreatic necrosectomy (1). After initial transgastric puncture of the fluid collection with a needle papillotony knife under $x$-ray screening control, we now use an over-the-guidewire technique to insert a 12 French pigtail catheter.

Five patients were treated successfully and their stents were remoyed at 3 months with repeat CT showing no reformation of the fluid collections. In one patient, the techrique failed due to inadequate adherence of a pseudocyst to the stomach wall. The cyst was punctured with the needle papillotome but during initial injection of contrast the cyst disnupted and an open cystojejunostomy was performed.

During the same time period, 12 other patients underwent open necrosectomy/ abscess drainage following acute pancreatitis and 3 underwent open drainage of pseudocysts associated with significant necrotic debris or poor adherence to the stomach wall. There were no hospital deaths.

We feel that endoscopic transgastric drainage of selected pancreatic pseudocysts and abscesses is a useful option in the managernent of these complications. It avoids laparotomy and allows earty hospital discharge. 
Polymorphism of the IL-10 gene in patients with acute pancreatitis

$K$ Sargen, AG Demaine, AN Kingsnarth

Postgraduate Medical School, Plymouth, UX

Interleukin - $10(\mathrm{LL}-10)$ is an important regulatory cytokine which suppresses the inflammatory response seen in acute pancreatitis (AP). Polymorphisms of the IL-10 gene promoter may influence IL-10 levels so affecting disease susceptibility and severity.

Aim To investigate if 3 polymorphisms at positions $-1117,-854$, and -627 in the IL-10 promoter region influence susceptibility to and severity of AP.

Methods The IL-10 locus was amplified by PCR from the DNA of 125 patients and 130 controls. Sequence specific oligonucleotide probing (SSOP) was then used to detect the 3 biallelic polymorphisms. Allelic and genotype frequencies were compared in $2 \times 2$ contingency tables using the chi-squared statistic.

Results 36 of 125 patients had severe disease (Atlanta), 18 had an organ failure score of $\geq 3$. AP was due to alcohol in 21 patients, gallstones in 64 , and was idiopathic in 34 . No difference was seen in allelic or genotype frequencies at any of the 3 loci between patient groups and controls, or patient groups stratified according to disease severity or aetiology.

Conclusion $\mathbb{L}-10$ promoter polymorphisms do not influence disease susceptibility or severity in AP.

United Kingdom and Ireland survey of antibiotic prophylaxis in acute pancreatitis.

JJ Powell, E Campbell, CD Johnson * A AK Siriwardena

University Departments of Surgery, "Southampton General Hospital \& Royal Infirmary of Edinburgh.

Introduction Although trials suggest benefits from the prophylactic use of antibiotics in severe acute pancreatitis, the results are inconsistent, giving rise to the potential for disparate clinical practice. This study therefore aimed to ascertain current practice relating to prophylactic antibiotics in acute pancreatitis.

Methods A postal questionnaire survey of 1103 members of the Association of Surgeons of Great Britain and Ireland was performed. Following exclusion of. surgeons not treating patients with acute pancreatitis, analysis was carried on 429 replies.

Results Respondents treated a median of 12 patients with acute pancreatitis per year. Prophylactic antibiotic therapy is used by $88 \%$. Of those using prophylactic antibiotics $24 \%$ used them in all cases. For surgeons professing selective use the indications are: prognostically severe $(81 \%)$, pancreatic necrosis $(65 \%)$, jaundice $(49 \%)$ and organ dysfunction (34\%). Cephalosporins are prescribed in $72 \%$ of cases, with cefuroxime the most common. Combination therapy with metronidazole is used in $48 \%$. Duration of therapy is a median of 5 days for $87 \%$ of respondents. An adverse drug reaction attributable to prophylactic antibiotics was reported by $11 \%$,

Conclusions Although therapeutic issues remain, this survey establishes that the prescription of prophylactic antibiotics in acute pancreatitis has become widespread. However there does not appear to be a consensus regarding the indications, type and duration of antibiotic prophylaxis. 
Endoscopic ultrasound (EUS) in the diagnasis of pancreatobiliary numours

SA Norton, D Alderson

Bristol Royal Infimary

Method Failure to visualise a pancreatobiliary tumour on imaging investigations, or difficulty in differentiation of tumour from focal chronic pancreatitis (CP) may reduce the chances of identifying a small numour when resectable. The value of EUS in diagnosing such tumours was assessed in 82 patients with suspected malignancy. EUS findings were compared to those of other investigations and to the final diagnosis.

Results A final diagnosis of neoplasia was made in 48 cases. Sensitivity of EUS in diagnosing tumours was significantly greater than that of transabdominal ultrasound (TUS) and computerised tomography (CT) $(p<000001$ and $p=0.04$ respectively). The sensitivity and specificiry of EUS in diagnosis of neoplastic disease when other investigations were normal was $92.3 \%$ and $95 \%$ respectively. Seven patients had tumours of $2 \mathrm{~cm}$ or less in diameter, visualised by EUS in $7 / 7$, TUS in $0 / 5$ and CT in $3 / 7$ cases. EUS was only marginally better than CT, ERCP or TUS in differentiation of neoplasia from focal CP.

Conciusion EUS is more sensitive than TUS or CT in diagrosing pancreatobiliary tumours, particularly when small and can confidently exclude significant pathology in other cases. Large studies are required to determine whether identifying tumours at an earlier stage could have an impact on mortality.

EUS does not reliably distinguish focal CP from tumour.

Serum isoamylase and lipase activities in HIV positive clinical categories.

MJMJ Deenamode, A Pomiak" \& RA Sherwood

Departments of Clinical Biochemistry and Sexual Health", King's College Hospital, London SES 9RS

Increasing numbers of HIV + ve patients on nucleoside analogue therapy with reported pancreatoxic side effects will result in increased monitoring of serum amylase which remains the cheapest and most widely used test in the UK. The patterns of serum total pancreatic amylase and lipase were investigated in a random population of 95 HIV +ve patients, 77 (81\%) males, mean age 34 years. The population was divided into clinical categories: asymptomatic $(A, n=23)$. symptomatic non AIDS $(B, n=49)$ and $A I D S(C, n=23)$. Comparison of each category with adult controls $(n=16)$ showed a tendency for lipase alone to be higher in category A, $p=0.03$ ( 16 whites, 6 blacks, 1 other), a tendency for total, pancreatic and salivary type amylase and lipase to be higher in category $B$ (33 whites, 16 blacks, 2 other) patients, $p=0.04,0.06,0.07$, and 0.03 respectively. Concomitant elevated pancreatic amylase and lipase were not observed in any of the patients. Comparison of results between ethnic groups revealed total and salivary type amylase to be higher in the black HV +ve population, $p=0.002$ and 0.001 respectively. In addition to ethric annylasaemia, contribution by salivary type amylase due to oral and oesophageal infections should be considered when interpreting elevated amylase results. 
Pancreatic lipase and amylase in fulminant bepatic failure: an immunohistochemical survey.

MJMU Deenamode, B Portmann", R Przemioslo', A Rayner \& RA Sherwood Department of Clinical Biochemistry and Institute of Liver Studies ${ }^{\circ} \cdot K^{\prime}$ ing's College Hospital, London

Pancreatic, salivary amylase, lipase and trypsin have been reported to be present in hepatic large bile ducts, septal ducts and peribiliary glands in post montem normal livers. The histological presence of amylase and lipase in fulminant hepatic failure and their possible contribution to serum levels were investigated by immunoreactivity of pancreatic amylase and lipase antibodies on 5 native and 1 graft biopsy, 4 hepatectomy and 3 post mortem liver specimens obtained from 11 adult patients and accompanied by measurement of serum total pancreatic amylase and lipase. Positive immunoreactivity with monocional pancreatic lipase antibody was observed in $12(92 \%)$ of the 13 samples. Positivity with monoclonal and polyclonal pancreatic amylase antibodies were observed in $2(15 \%)$ and $7(54 \%)$ liver samples. immunoreactivity was generally observed periportally, in surviving parenchyma, isolated cells and in regenerating cells. The presence of pancreatic lipase in regenerating hepatic cells suggested a possible developmental morphogenesis with the pancreas or a role in the regeneration process. Furthermore, cholestasis and coinciding elevated serum pancreatic lipase suggested hepatic synthesis, storage or impaired clearance of the enzyme.

Serum isoamylase and lipase activities in preterm infants.

MJMJ Deenamode, RM Nicholl", HR Gamsu' \& RA Shenwood

Department of Clinical Biochemistry and Children Nationwide Neonatal Unit" King's College Hospital, London

Previous authors have reported that amylase and lipase activities were almost undetectable in the newborn infant and that exocrine pancreatic function is compromised by intrauterine growth retardation (IUGR). Investigation of possible factors influencing exocrine pancreatic function in a group of preterm infants was carried out by measurement of serum pancreatic lipase (Sigma Diagnostics, UK), total and pancreatic amylase (Roche Mannheim Diagnostics, UK) activities in 70 pretern neonates aged between 0 and 30 days, median birth weight $1193 \mathrm{gm}$ (range 610 to 1890 )

Serum lipase and pancreatic amylase was undetectable in $1 \%$ and $89 \%$ of neonates, respectively. $47(67 \%)$ neonates had serum lipase in the adult reference range, median 25 IU/L (range 16-74).

Furthermore, $9(13 \%)$ had lipase activities above and $14(20 \%)$ below the adult range. No differences were noted comparing inclusion and exclusion of neonates fed on raw human milk or IUGR. Selective exocrine pancreatic expression of lipase but to a much lesser extent pancreatic amylase would correlate with fat being the main source of energy at birth. The possibilities of identifying those infants with reduced lipase levels could have implications for enteral nutrition of such babies. 
Audit of deaths from acute pancreatitis in the south east of Scotland 1995-96: Evidence for variation in management?

Aims and Method: An audit of deaths under surgical care from severe acute pancreatitis reported to the Scottish Audit of Surgical Mortality (Edinburgh Office) during 1995-96.

Results: 61 deaths were reported: median age was 70 years (range 31-91) with 13 patients (21\%) under the age of 55 years. The predominant mode of death was multipie organ failure ( 49 patients). The median time from admission to death was 8 days: 20 patients ( $33 \%$ ) died within the first $\mathbf{4 8}$ hours. 35 patients were managed in specialist units, 14 of whom had been transferred from other units. In 3 patients, delay in transfer was thought to contribute to their death. While pulmonary embolus contributed to death in 3 patients, DVT prophylaxis was not used in 29 patients $(48 \%)$ ). 40 patients $(66 \%)$ were admitted to the ICU. There were no ICU beds available for 3 patients and a decision to limit treatment was made for $B$ patients. No reason was given for the remaining 10 patients whose ages ranged from 75-89 years.

Conclusion: A third of patients dying from acute pancreatitis rapidly develop MOF and die within 48 hours of admission. Some patients were either not transferred to specialist units or were transferred late; DVT prophylaxis was not universal; ICU facilities were withheld from a number of elderly patients.

Investigation of the effect of hypoxia on matrix metalloproteinase expression in pancreatic cancer cell lines

Williams DM, Griffin JA, Matthews GM Buckels JAC, Bramhall SR.

Department of Surgery, University of Birmingham

Tumour growth is limited by the availability of oxygen and growth beyond a few hundred micrometres requires neovascularisation. Access to the systemic circulation is required for metastasis. The matrix metalloproteinases (MMIPs) play a critical role in metastasis and angiogenesis and hypoxia is a potent stimulator of angiogenic factors and may increase invasion.

The aim of this study was to investigate the effects of hypoxia on MW expression in three pancreatic (BxPC3, Pancl, SUTT-2) and one fibrosarcoma (HT-1080) cell lines. Oxygen tensions of $20 \%, 5 \%, 1 \%$ and $0 \%\left(5 \% \mathrm{CO}_{3}\right)$ were investigated. MMP expression profiles of all cell lines at all oxygen tensions were determined by RT-PCR and the level of mRNA quantified by multiplex PCR. MMP protein levels were examined by westem blorting. Expression and activity of MMPs were investigated by zymography and a fluorogenic substrate assay.

Protein analysis was not sensitive enough to detect differential expression but PCR analysis identified several MMPs which were upregulated by hypoxia. The greatest upregulation was seen in SUTT-2 cells with Stromelysin 2, MTMMP2 and MTMMP4 most affected. MTMMP2 and MTMMP4 mRNA levels showed a several hundred fold increase at $1 \%$ oxygen.

In conclusion hypoxic regulation of MMP expression may play an important role in angiogenesis and tumour invasion. 
The evolution of a system specific tumour database

K Le Camu, BK Gunson, SR Bramhall. DF Mirza, AD Mayer, P McMaster, JAC Buckels

Liver and Hepatobiliary Unit, Queen Elizabeth Hospital, Birmingham B15 2TH

The Bristol enquiry led to a requirement that all units return data on operative outcome, but the quality of the collected data is left up to individual units. This unit has designed a hepatobiliary database, but guidelines introduced since its inception has led to several developments in its design.

The database bas been developed to build a risk stratification model for operative patients and a standardised approach to reporting to allow inter-unit comparison. Data are collected prospectively in outpatients and from medical, nursing, $x$-ray and clerical staff, by a dedicated database co-ordinator.

Data collection started on 1/9/97 and 551 patients have been entered, 65 with pancreatic adenocarcinoma, 13 ampullary carcinoma and 36 chronic pancreatitis. 37 patients with cancer underwent resection ( 30 day mortality $=5 \%$ ) and 21 had a palliative bypass ( 30 day mortality $\Rightarrow 9.5 \%$ ). A further 12 patients had no surgery and were entered into clinical trials. Data will be presented on patient demographics and tumour pathology. From 1/9/98 additional data will be collected on risk factors in patients undergoing surgery which will allow stratification when companing results between units. Standardised proforma have been developed for histopathological, radiological and operative reporting in pancreatic tumours and are being evaluated.

Accurate data collection is now mandatory and these data could be used to disadvantage units. The establishment of a well designed, adequately staffed database has allowed the development of a flexible system that can be easily modified and allows the collection and analysis of data for inter-unit comparison.

Neutrophil activation in acute pancreatitis - the "two bit" hypothesis coufirmed

A Steven, CMCKay, S Robertson, S Evans, CR Carter, CW Imrie

Lister Dept of Surgery, Glasgow Royal Infirmary

Background: Early organ failure is an important source of morbidity and mortality in acute pancreatitis and there is evidence that it is mediated by activated inflammatory cells. It has been proposed that this process may occur in two stages: an initial priming of inflammatory cells followed by a further inflammatory "hit" which causes further cell activation, inducing organ damage.

Methods: Patients with acute pancreatitis who did not have organ failure on admission (as defined by Atlanta criteria) had daily sampling of peripheral venous blood. Neutrophil activation stanus was assessed by whole biood chemiluminescence.

Results: On admission to hospital, neutrophils from patients with acute pancreatitis had a significantly greater oxidative burst than those from control patients whether or not organ failure developed. In the group of patients who developed organ failure, there was a significant increase in respiratory burst over subsequent days which was not observed in patients without organ failure.

Conclusion: The development of early organ failure in acute pancreatitis is associated with activation of previously primed neutrophils, suggesting a further inflammatory stimulus or "second hit". 
Recent time trends in England and Wales in gastric and pancreatic cancer mortality

D Fitzsimmons, C. Osmond, S George, C.D Johnson

Pancreatic and Biliary Research Group. University of Southampton

Aims: Time trend analysis provides an important insight into the evolution of aetiological risk factors in disease. We have examined mortality rates in England and Wales over 1950-1994 to observe any age-period-cohort effect on current trends.

Materials and methods: Mortality data was collected from the Office of National Statistics over the time periods. The age-period-cohort effect was modelled using Poisson Error.

Results: Pancreatic cancer rose from 7.9 per 100,000 to a peak of 12.3 per 100,00 in the mid 1980s in men. Since then, there has been a slight downwards trend. In women, the mortality rate has risen from 6.5 per 100,000 to 12 per 100,000 in the late 1980s. Since then, mortality rates have stabilised. In gastric cancer, mortality rates have shown a decrease, from 37.9 per 100,000 to 19.6 per 100,000 in 1990-94 (men) and 27.8 per 100,000 in 1950 to 12.2 per 100,000 in 1990-94. Like pancreatic cancer, these trends are seen in the older age groups. When period and cohort effect is modelled, there is a downwards trend seen in pancreatic cancer for men and women. In gastric cancer, there is no period effect but a significant cohor effect in both sexes $(p<0.05)$.

Conclusion. The period effect in pancreatic cancer is parallel, but occur later than other disease series (e.g. lung cancer) and suggests a possible link with declining rates of tobacco consumption. In gastric cancer the downwards trend and cohor effect in both sexes may possible be explained by changes in prevalence of Helicobacter Pylori.

Minimal access techniques in the management of infected pancreatic necrosis R Carter, CJMCKay, CWImrie

Lister Department of Upper GI Surgery, Glasgow Royal Infirmary

Infected pancreatic necrosis traditionally requires initial radical debridement with continuous post-operative peritonea] lavage, the majority requiring more than one laparotomy. Such surgery in patients with established multi-organ dysfunction is associated with a mortality approaching $20 \%$. We describe our initial experience of primary percutaneous debridement of pancreatic necrosis and the use of sinus tract endoscopy in the management of residual sepsis.

Methods: Following CT guided access and graduated dilatation, we used an operating nephroscope to allow irrigation, suction and piecerneal clearance of the necrotic solid material from the abscess cavity. A twin lumen drainage system was then inserted to allow continuous post-operative lavage. Subsequent sepsis. was. managed by Sinus Tract Endoscopy and further postoperative irrigation-

Results: All patients had bacteriologically proven retroperitoneal sepsis secondary to acute pancreatitis. To date we have managed 7 patients with primary percutaneous debridement, and 10 with sinus tract endoscopy, five of whom had undergone an initial open procedure. One patient required conversion for haemontrage. Two patients died, one from multiorgan failure, and one from liver failure one month after resolution of their sepsis. Three are currently inpatients. All but two were managed within the HDU. The remainder have been discharged, one of whom required late endoscopic drainage of a pseudocyst.

Conclusion: Our initial results are encouraging, and we are currently undertaking a more in-depth appraisal of the technique. 